\title{
Computational studies of a DNA-based aptasensor: toward theory-driven transduction improvement.
}

Mario Araujo-Rocha ${ }^{1}$, Benoît Piro ${ }^{1}$, Vincent Noël${ }^{1}$, Florent Barbault ${ }^{1 *}$

1. Université de Paris, ITODYS, CNRS, F-75006 Paris, France

*Corresponding author email: florent.barbault@u-paris.fr

A preliminary version of this work was deposited in ChemRxiv

\section{Abstract}

Aptamers are a class of bioreceptors intensively used in current analytical tools dedicated to molecular diagnostics due to their ability to perform large structural reorganization upon target binding. However, there is a lack of methodologies allowing to rationalize their structure in order to improve the transduction efficiency in aptamer sensors. We choose here, as a model system, a three-strand DNA structure as probe, composed of two DNA strands anchored on a gold surface and partially hybridized with an aptamer sequence sensitive to Ampicillin (AMP). The DNA structure has been designed to show strong structural change upon AMP binding to its aptamer.

Using a set of computational techniques including molecular dynamics simulations, we deeply investigated the structure change upon analyte binding, taking into account the grafting on the surface. Original analyses of ions distributions along the trajectories unveil a distinct pattern between both states which can be related to changes in capacitance of the interface between these states. To our knowledge, this work demonstrates for the first time the ability of computational investigations to drive, in-silico, the design of aptasensors.

Keywords: Molecular Dynamics, EGOFET, Biosensors, Aptamers, Biointerfaces, Ampicilin, DNA.

\section{Introduction}


Aptamers are structured DNA or RNA sequences selected in-vitro to bind specifically to a non-nucleic target ${ }^{1}$. Current SELEX (systematic evolution of ligands by exponential enrichment) methodologies allow to identify quickly specific aptamer sequences against a broad variety of targets e.g., from whole cell to low molecular weight molecules. Aptamers have proven their relevance as specific bioreceptors and used to date in many highly sensitive analytical methodologies ${ }^{2,3}$. Many of them, named aptasensors, involve the anchoring of one of the aptamer ends to a surface acting as the transducer element ${ }^{4}$. Among the varieties of aptasensors, those that do not require labelling of the distal end of the sequence (i.e., by a biomolecular probe, a protein, a nanoparticle, etc.) to assay the target constitute the most interesting ones according to their ease of use and their often better analytical characteristics. This is especially true for low molecular weight targets that can show dramatic affinity decay upon even minor aptamer labelling ${ }^{5}$. To avoid labelling, powerful transduction strategies have been proposed, those based on the monitoring of the charge distribution change close to the surface upon target binding being particularly promising.

Nucleic acids sequences are polyelectrolytes and their conformational change associated to the target binding can lead to charges reorganization close to the transducing surface. Current analytical techniques such as surface plasmon spectroscopies and field-effect transistors can detect small ionic distribution changes of an electrolyte. The intensity of the recorded signal depends on the refraction index and on the capacitance of the thin solution layer close to the surface, respectively. These techniques have already shown their relevance in the field of aptasensors ${ }^{6,7}$. However, the inherent lack of affinity of aptamers selected against low molecular weight targets, with affinity constants usually in the $\mu \mathrm{M}$ range, requires innovative amplification strategies to reach lower limits of detection.

Recent advances in designing functional DNA architectures ${ }^{7,8}$ allow to consider a new class of highly sensitive aptasensors that switch their entire conformation even if only few bases are involved in a target binding process. In most of the cases, the aptamer sequence is introduced inside a host matrix (scaffold) participating in its overall 3Dstructure. ${ }^{9}$ By properly designing the nucleic acids scaffold i.e., when anchoring aptamer functions to the scaffold are the same as those interacting with the target, scaffold structure change may be induced by the target binding. The equilibrium constant of the 
competitive exchange reaction involving the aptamer sequence, the scaffold and the target must thus be carefully tuned in order to maintain both a scaffold stability at ambient temperature and a relevant apparent aptamer/target affinity constant.

To achieve the design of such structures, information about the structural mechanism of the biomolecular complex formation is required. However, precise aptamer/target complex structure is known only for a few systems. In these well-described cases, the aptamer is already folded to bind the target constituting a relevant starting point for the overall structure determination ${ }^{10,11}$. However, in the most general case, "induced-fit mechanism"12,13,14 is involved and the structure cannot be single-handedly deduced from classical tools such as mfold alone. Besides, the vast majority of biosensor devices requires immobilization of a biomolecule on a surface which involves surface/biomolecule interactions and thus structural modifications. Despite its wide importance, experimental investigations of such structural modifications remain elusive ${ }^{15}$ so that molecular simulations, in this context, represent an alternative to understand the underlying physical processes which occur at the biomolecule/surface interface ${ }^{15}$.

The available literature on molecular modeling studies of sensing architectures including an immobilized bioreceptor is scarce. Some recent articles deal with peptide-based systems (i.e., ochratoxin ${ }^{17}$, carboxyhemoglobin ${ }^{18}$, dopamine ${ }^{19}$ ) but very few engage a nucleic acid sequence as a receptor. Choodet et al. deciphered the mechanisms of naked-eye aptasensors, based on citrate-capped gold nanoparticles (AuNPs), for 8-oxo$\mathrm{dG}$ detection, using molecular dynamics (MD) simulations ${ }^{19}$. The modeling of the most encountered aptasensors format where the nucleic acid sequence is immobilized onto a solid, was only discussed, to our knowledge by Ruan et al. They analyzed the effects of adding T6 linker to an anti-flavin RNA-based aptamer and its anchorage to a 1-mercapto6-hexanol-functionalized gold surface on the biorecognition process ${ }^{20}$. They concluded that the immobilized aptamer undergoes a structural reorganization, which may cause a loss in affinity, and that this restructuring is due to an anisotropic ion distribution around the aptamer. This loss of affinity is dominant when immobilizing bioreceptors to a surface, which is well-known experimentally ${ }^{21}$. Our aim is here to take advantage of this ionic distribution variation, at the vicinity of an aptamer grafted on a gold surface, to create an efficient biosensor. 
In this work, we propose a DNA-based biorecognition structure immobilized on a gold surface taking advantage of a conformational switch to promote a change in the ionic concentration at the vicinity of the gold surface, which can be a label-free method of transduction for a future EGOFET-based biosensor. The recognition process is mediated by the presence of an aptamer sequence available for interacting with the target molecule. As a proof-of-concept, we have chosen ampicillin (AMP) as our molecule of interest, which is environmentally important as it plays an important role in bacterial antibiotic resistance ${ }^{22-24}$. As aptamers can be readily developed for a large group of molecules, we believe that the results obtained with this proof-of-concept would be straightforwardly adapted to other similar analytes.

\section{Computational Details}

\subsection{In-silico constructions}

DNA systems were built with the nucleic acid builder (NAB) module of Amber software ${ }^{24}$. Manual editions were performed to generate the sequences displayed in Schematic 1. The initial aptamer hairpin structures were obtained with the help of the Mfold ${ }^{26}$ platform and 3D RNA Web server ${ }^{27}$. The gold surface model used in this work is the Au\{111\} functionalized with a 6-mercapto-1-hexanol self-assembled monolayer as already defined and validated in a previous work on aptasensor simulations ${ }^{20,28}$. This surface is of size $9.1 \times 8.9 \mathrm{~nm}^{2}$, large enough to encompass the off or on-state. The AMP compound (Figure $\mathrm{S} 2$ in the supplementary material) was generated with the help of the maestro Schrödinger program ${ }^{29}$. This last software was also used to graft the concerned DNA sequences on the gold surface in combination with the xleap module of AmberTools.

AMP docking computations were performed with the Autodock 4.2 software ${ }^{30}$ specifically modified with the help of an artificial intelligence procedure, to cope with nucleic-acid targets $^{30}$. This software modification has already been validated and proven its reliability ${ }^{31-33}$. The Lamarckian Genetic Algorithm was employed ${ }^{34}$ for the global optimum binding position search. For each DNA aptamer conformers, grid boxes enough to encompass the whole target structures were created around the DNA conformers, so that the AMP ligand may interact on any target location without a priori. 100 docking poses were realized for each target and the resulting ligand structures were then clustered according to a RMSD (root-mean-square deviation) lower than $2 \AA$. The conformation 
selected was the one which presented the best docking score in the most populated cluster ${ }^{34}$.

\subsection{MD simulations}

All molecular dynamics simulations (MD) were realized with the Amber $20^{36}$ software suite under periodic boundary conditions with Particle Mesh Ewald (PME) method ${ }^{37}$. Systems were solvated using TIP3P water molecules ${ }^{38}$ and ionic concentrations were adjusted to $160 \mathrm{mM}$ with $\mathrm{NaCl}$ in order to fit usual experimental conditions. The OL15 force-field ${ }^{39}$ was used for DNA and AMP was considered with the general amber force field ${ }^{40}$ (gaff). For this last ligand, atomic partial charges were derived through AM1-BCC method 41,42 as its reliability has been demonstrated ${ }^{43}$. Consideration of the gold surface functionalized with a 6-mercapto-1-hexanol self-assembled monolayer were reproduced from previous works of the team ${ }^{20,28}$. MD simulations started with a first minimization before engaging a MD heating phase of 150 ps in order to reach $300 \mathrm{~K}$; this last temperature was chosen for all MD simulations. A molecular dynamics in NTP conditions was then engaged for 500 ps in order to equilibrate the solvent density. Then the simulation systems were switched to the NVT ensemble, more suitable for simulations with a solid/liquid interface $^{15}$. MD productions (50 ns to $200 \mathrm{~ns}$ ) were made with the help of the SHAKE algorithm ${ }^{44}$ in order to use a time increment of $2 \mathrm{fs}$.

\subsection{Trajectory analyses}

VMD software ${ }^{45}$ was used for trajectories visualization and figures generation while other analyses were performed with the cpptraj module of AmberTools ${ }^{46}$.

Free energies of binding between AMP ligand and its aptamer were evaluated with the MMGBSA technique ${ }^{47}$ for which we already demonstrated its reliability in various contexts of ligand associations ${ }^{47-49}$. These last computations were made over the last 50 ns of trajectories. Free energy landscape was deduced from trajectory analyses by using Boltzmann statistical mechanics as previously reported by Ruan and collaborators ${ }^{20}$.

\section{Results and Discussion}

\subsection{Aptasensor description}


Our system has been designed while keeping in mind its experimental feasibility. Alkylthiol self-assembly on gold ${ }^{50}$ is one the most popular method to immobilize a DNA structure in aptasensor. Grafting can be easily done using 5' or 3' alkylthiol-modified DNA sequences. Moreover, saturation of the gold surface with a short alkyl-thiol such as mercaptohexanol $(\mathrm{MCH})$ is commonly realized in order to displace occasional impurities and avoid the collapse of biomolecules onto the uncovered surface. As a consequence, we investigated the structure depicted on Figure 1.

The supramolecular DNA structure is composed of two sequences immobilized on a gold surface through Au-S bond and bridged through a third strand that contains the anti-AMP aptamer sequence at one of its hybridized extremities (off-state, see Fig. 1 left). The presence of AMP in the media is expected to trigger the de-hybridization of the aptamer sequence in order to fold with the ligand leading to another supramolecular structure (onstate, see Figure 1 right).

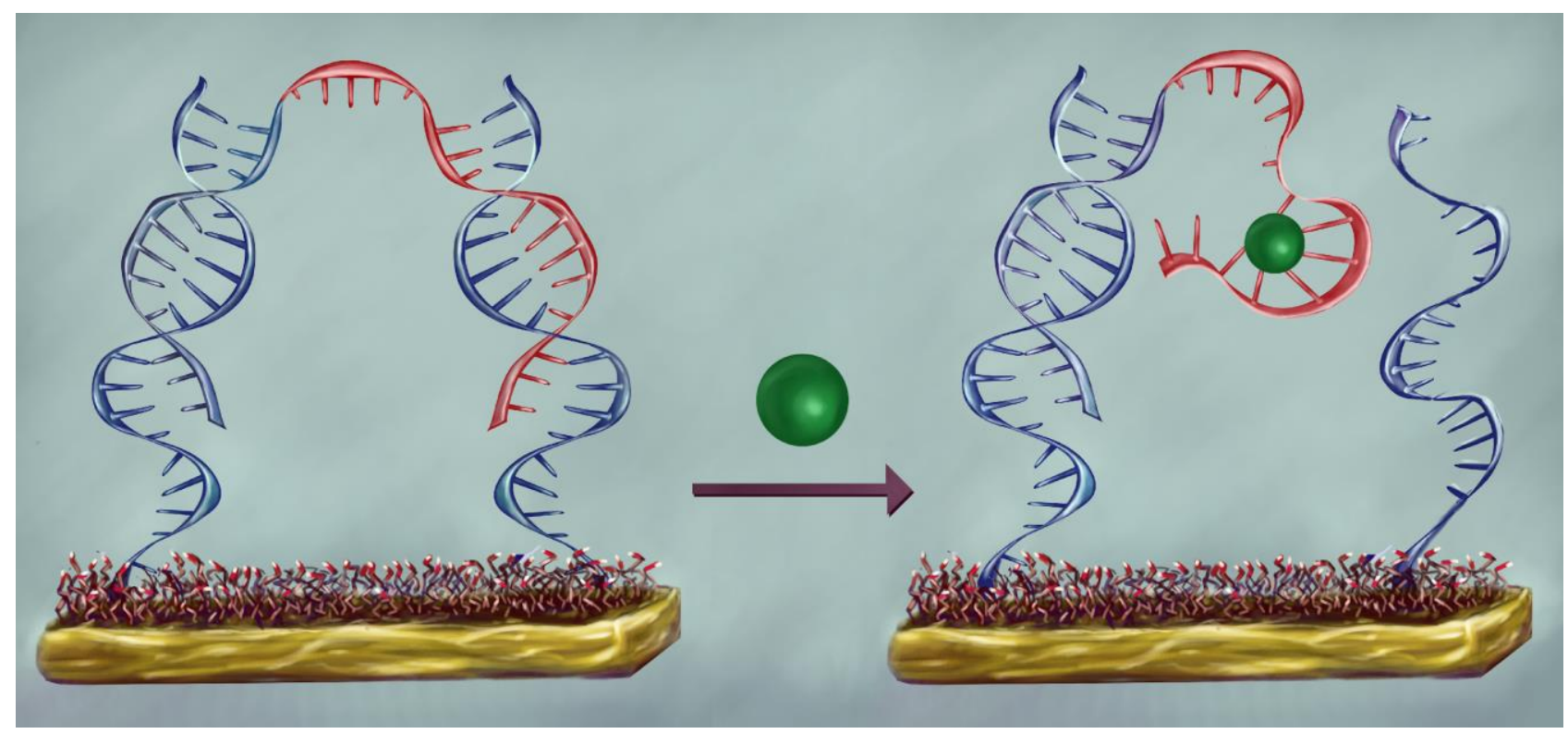

Figure 1. General scheme of the functioning of the proposed system, passing from an off-state structure (left) to an on-state structure (right) in the presence of the target molecule (AMP) (green), by the formation of the aptameric hairpin (red).

\subsection{Anti-AMP aptamer structure}

Inspired by the work made by Song and collaborators ${ }^{51}$, we designed the 70-bases DNA three-strand system presented on Schematic 1a). The structure is composed of a central 33-mer sequence containing the anti-AMP sequence, hybridized by 13 base pairs on 
each extremity to two other sequences 18- and 19-base long which form the two legs grafted to the surface through a $\mathrm{C}_{6} \mathrm{H}_{12}-\mathrm{SH}$ function.

One key element of our study is the analysis of the capacity of this nucleic acid to achieve the expected arch organization after we graft the thiolated extremities to the surface. We desire that the nucleic acid structure attains a stable U-shaped folding where both extremities point towards the same direction in space, which would translate in a direct grafting on the surface. Consequently, in the following, the U-shaped geometry of the DNA will be a noteworthy element of our structural enquiries since it allows a direct immobilization of our nucleic acid onto the gold/MCH surface.

Prior to immobilizing this system on a functionalized gold surface, we thus investigated the organization of this nucleic acid structure in isotropic conditions. The triple-strand structure contains distinct areas of various flexible behavior, being composed of two double-helix sections connected to a central single strand of nucleic acid. When we consider that a single strand DNA is overall more flexible than a double-helix, it is reasonable to consider that the central part, which bridges the two double-stranded structures, has the most important role on the curvature of the system. Therefore, we first decipher the conformational space of this central section i.e., the seven unpaired bases with four base-pairs on each side forming a 23-base system (see Schematic 1b)).

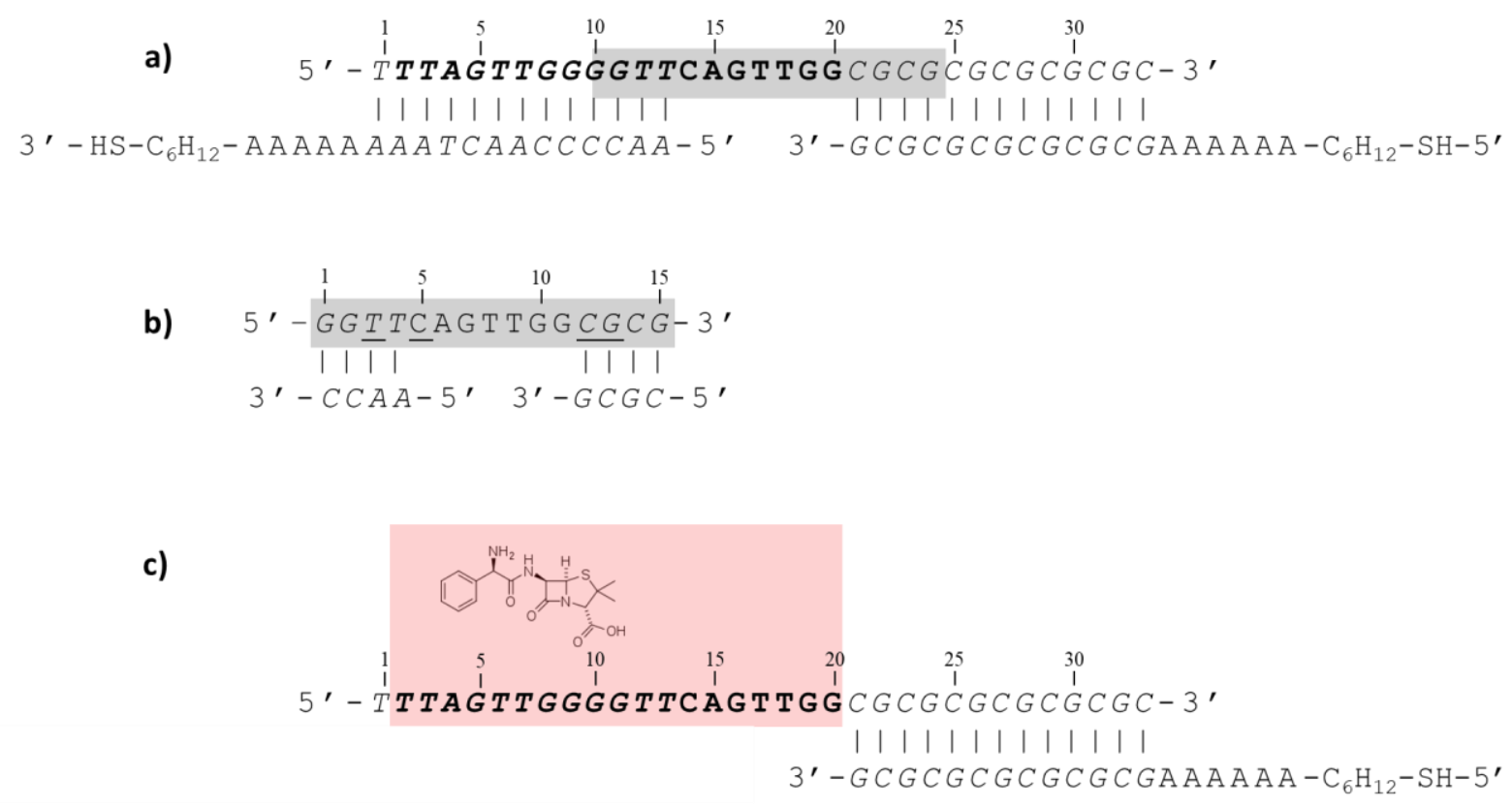

Schematic 1. a): 70-bases DNA three-strands system, (bold) anti-AMP sequence, (italic) bases involved in hybridization, b): a smaller DNA structure formed by the central section of the 
bridge sequence a), (underlined) bases involved in the analyses of interatomic distances performed, c) Structure of the off-state.

We ran six replicas of MD simulations in explicit solvent for the b) system. To elucidate the representative structure of the b) system and thus the structural organization of the "arch" with its "two legs", i.e., the de-hybridized versus the double-strands parts of the nucleic acids, a free energy landscape was computed from the molecular dynamics trajectory. Two distances, D1 and D2 corresponding to the distance between the phosphorus atoms of the nucleotides $\mathrm{C} 5 / \mathrm{C} 12$ and T3/G13 respectively were employed as coordinates for the free energy map. Only the last $100 \mathrm{~ns}$ of the 6 molecular dynamics replicas were used leading to a statistical view of $600 \mathrm{~ns}$. This resulted in the free energy landscape shown on Figure 2.

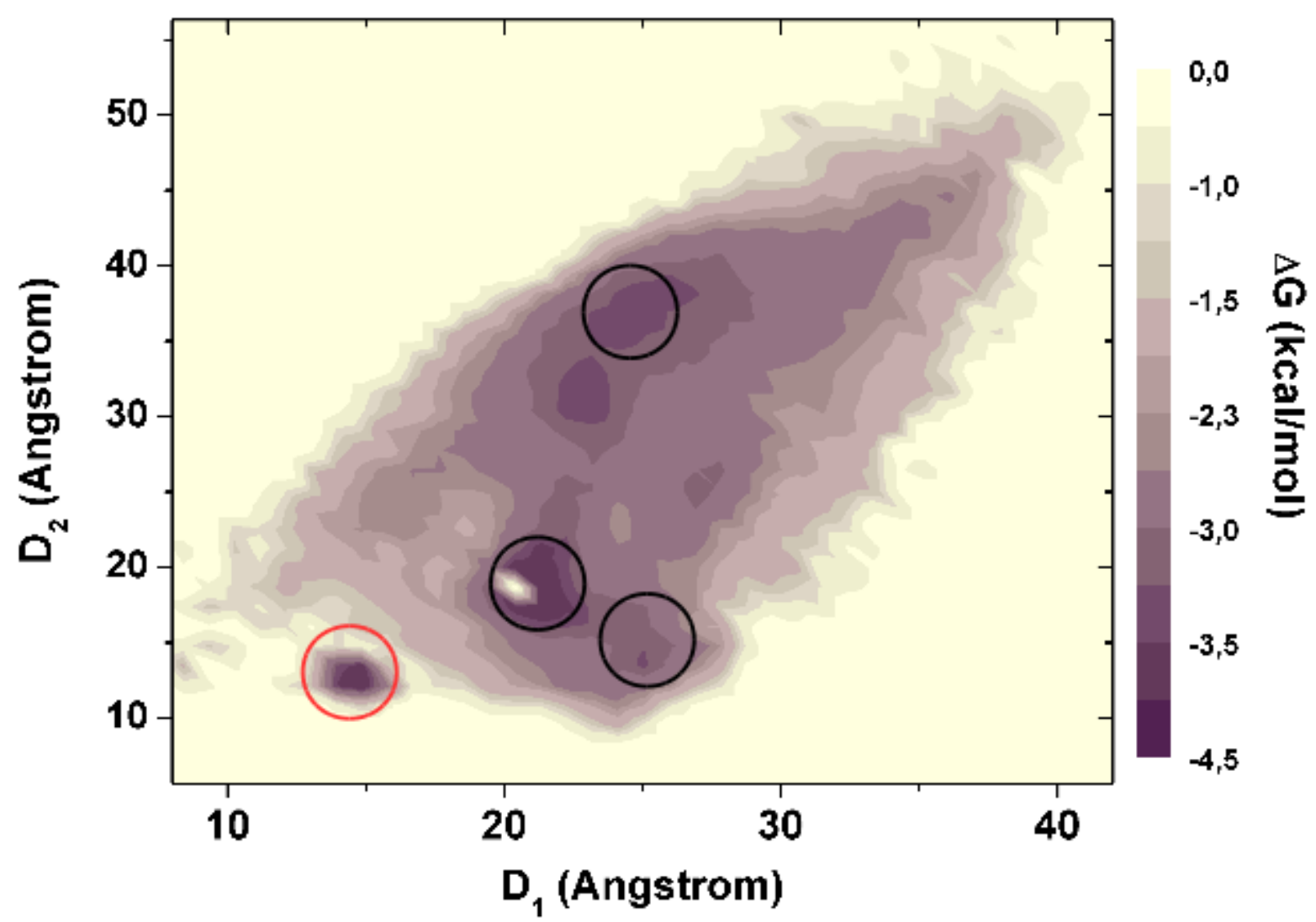

Figure 2. Free energy map of b) system with the local minima tested circled in black and the local minimum where we found our U-shaped structure circled in red. D1 and D2 are interatomic distances defined in the text of the underlined bases in the Sequence shown in 1b).

Figure 2 shows a large zone in the free energy landscape that can be easily accessible, showing that b) system is highly flexible. We were mostly interested in the energy minima, 
as they would be representative of the most stable structures. Amongst the minima, we actively looked for the one that would give us a U-shaped global structure when transposed back to the initial DNA sequence.

To get back to the structure of the full 70 bases system a) defined on Schematic 1, the values for D1 and D2 were then used as restraints $\left(5 \mathrm{kcal} . \mathrm{mol}^{-1} . \AA^{-1}\right)$, taken from the minima highlighted in circles on Figure 2. After 20 ns of MD simulations with restraints followed by 50 ns of relaxation, an appropriate U-shaped structure was extracted from the simulation using D1 between $13.50 \AA$ and $15.50 \AA$ and D2 between $12.00 \AA$ and 15.00 $\AA$ (area circled in red on Figure 2). Illustrations of all structures obtained in this process are available in the Supporting Information. It can be pointed out that despite the weak restraints force constant, the whole structure readily adapts to the restraints emphasizing the stability of this U-shaped structure. This last geometry was then grafted through a phosphate linker to the $\mathrm{Au} / \mathrm{MCH}$ surface on both extremities, forming the off-state bridge system shown on left part of Figure 3.
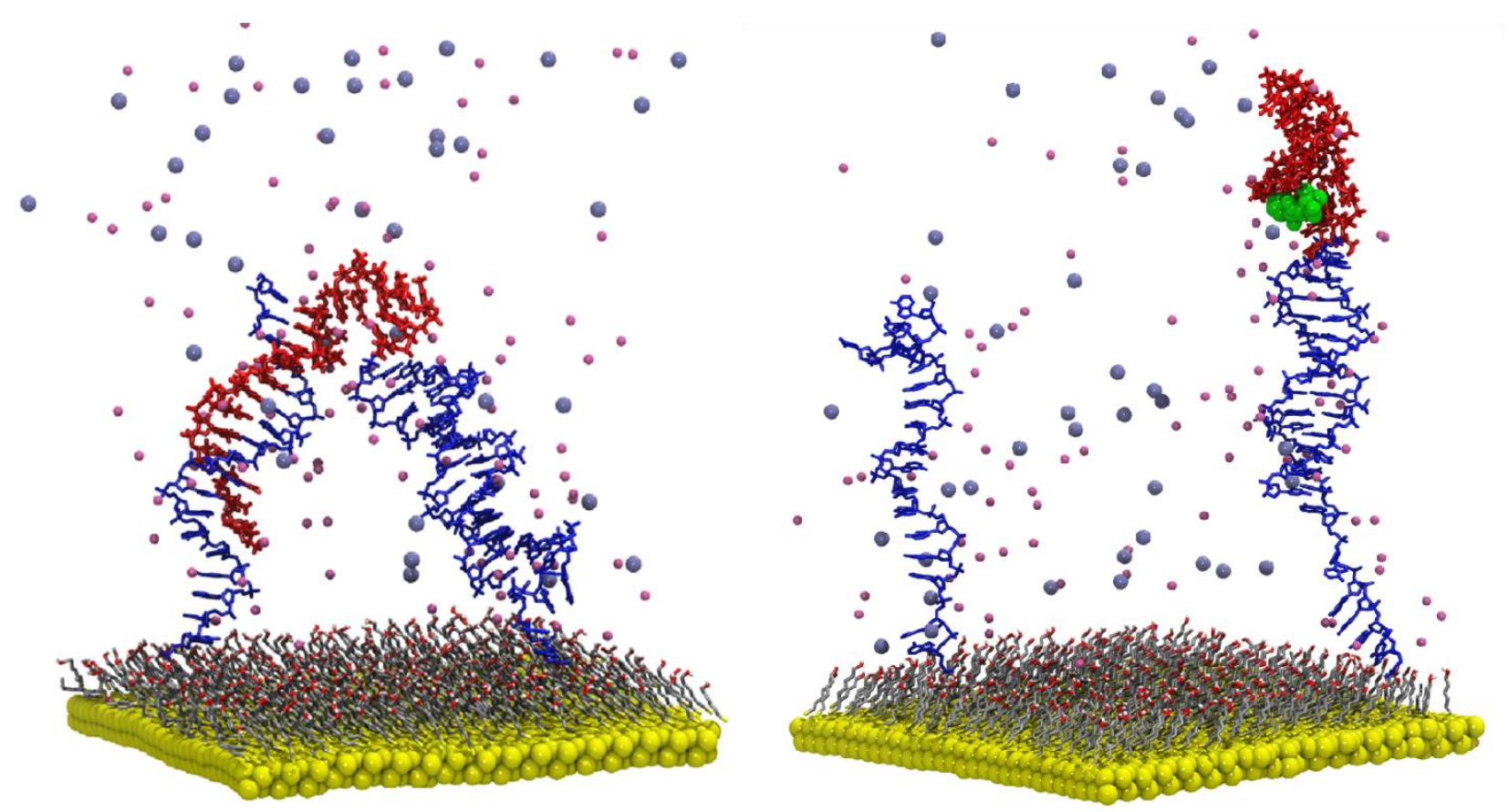

Figure 3. The complete off-state bridge system (Left) and open on-state system (Right) grafted to the $\mathrm{Au} / \mathrm{MCH}$ surface, with $\mathrm{Na}^{+}$(pink) and $\mathrm{Cl}^{-}$(purple) ions in solution and the AMP molecule in green. The aptamer sequence in both systems is marked in red. For the sake of clarity, water molecules are not displayed.

\subsection{Construction of the on-state system}




\subsubsection{Structure of the aptamer-AMP complex}

In order to build the on-state system bound to AMP, we first elucidated the structure of the aptamer-AMP complex. To date, there is no experimental 3D structure of this aptamer-AMP complex. The only available description corresponds to three sequences of AMP aptamers grafted on magnetic beads and used in a fluorescence-colorimetric strategy ${ }^{52}$. The prediction made with the mfold software of the $2 \mathrm{D}$ organization of these three DNA sequences ${ }^{24}$ unveil, in all cases, a hairpin organization in accordance with the experimental results found by Song and coll. ${ }^{51}$, shown on Figure 4a). From these results, we selected the sequence shown on schematic 1 (bases in bold), which according to their results, has the best binding interaction when bound to a surface. To obtain the 3D structure of the AMP aptamer, a computational pipeline method ${ }^{52}$ was undertaken (see Figure 4). This recent strategy has been specifically created for single-strand DNA aptamers and validated by reproducing numerous experimental structures ${ }^{52}$. The methodology consists in converting DNA into RNA prior to making explorational folding and then transforming back RNA into DNA.

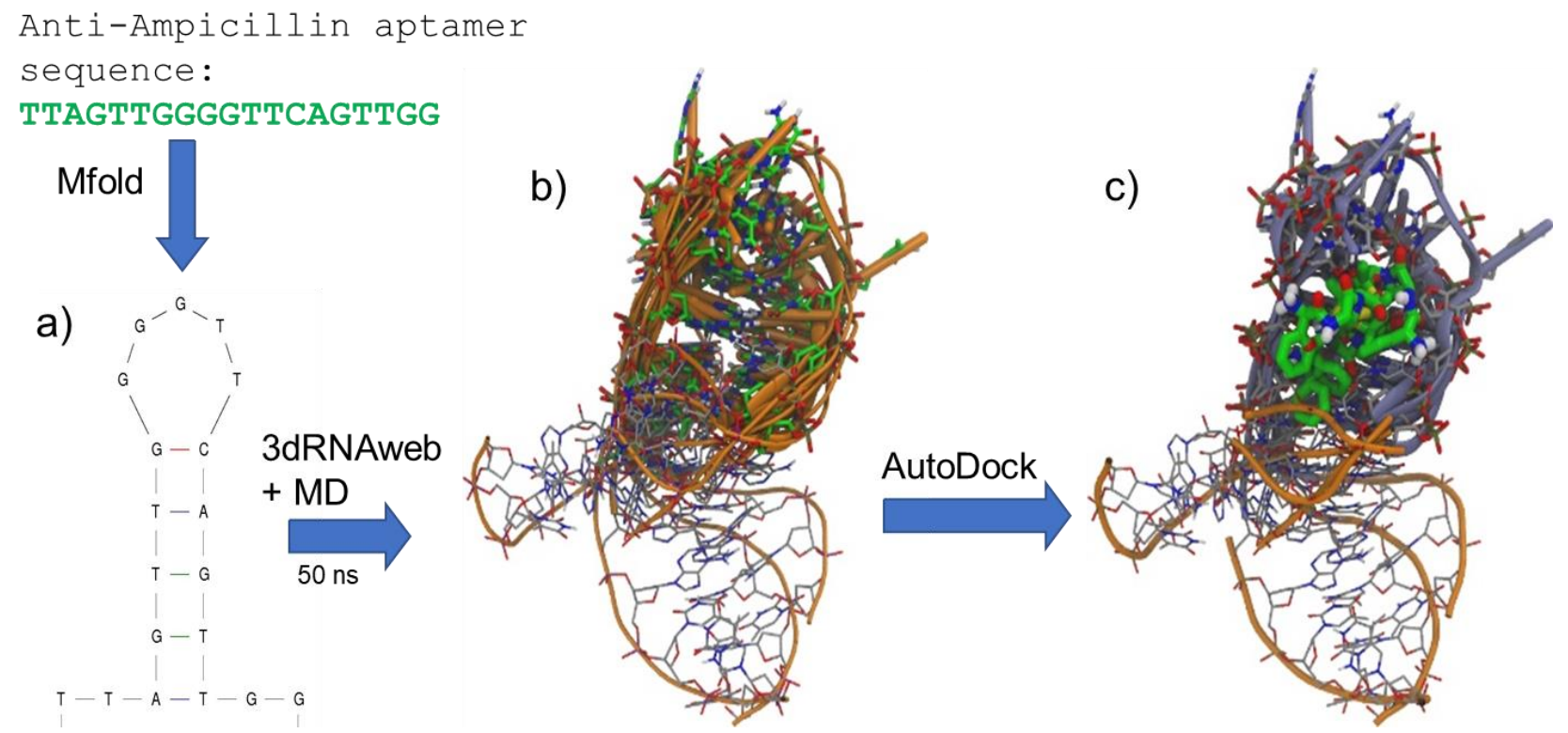

Figure 4. Computational protocol employed for the prediction of the most relevant threedimensional structure of the AMP aptamer: a) Predicted hairpin structure obtained from mfold;

b) Overlap of the five 3D DNA structures after prediction with 3dRNAweb and 50 ns of relaxation; c) Docking results of the five structures with the AMP molecule in green. 
The first step of this protocol is the generation of a 3D structure from its sequence. We selected the five best scored aptamer structures from 3dRNAweb and engaged a molecular dynamics simulation in a water box for $50 \mathrm{~ns}$ to relax these structures. At the end of this step, the five structures tended to converge to a consensual organization as shown on Figure 4b). To ensure that these 5 aptamer structures are able to bind AMP, molecular docking computations were engaged. The interacting site for the AMP molecule was defined as the whole hairpin so that the ligand is free to interact with the entire nucleic acid. Molecular docking energies, compiled on Table S1, cannot discriminate the 5 structures since their variations are below $2 \mathrm{kcal} / \mathrm{mol}^{34}$.

One common binding cavity is found for the 5 aptamer structures, as shown on Figure 4c) even though this ligand could interact with any other region of the DNA structure. On the work of Song et $a^{\not 1}$, the authors have compared several DNA sequences of AMP aptamers and speculated that a common sequence of GGTT in the vicinity of a GC basepair could be the interacting site of the AMP. In our 3D models, we observe that this pattern belongs to the DNA cavity where the AMP interacts, supporting the relevance of our structures. While the interacting cavity on the hairpin is roughly similar for all aptamer structures, their interacting modes evidence the AMP interacting with this cavity in different orientations on each structure.

To definitely state on the molecular structure of the aptamer, supplemental molecular dynamics simulations for 100 ns in water boxes were realized for the five complexes. From the five structures, only two kept the AMP in place over $100 \mathrm{~ns}$, both shown on Figure 5. We computed the binding energy using the MM-GBSA method and found two favorable energies of $\Delta \mathrm{G} 1=-35.6 \pm 4.0 \mathrm{kcal} . \mathrm{mol}^{-1}$ and $\Delta \mathrm{G} 2=-41.4 \pm 6.2 \mathrm{kcal} . \mathrm{mol}^{-1}$ for structures 1 and 2, respectively. Despite the fact that the free energies are, according to the recovery of uncertainty, of similar values, the Structure 1 was selected since a supplemental hydrogen bond is formed between the aptamer and AMP and the conformation of the amide bond in AMP (see Figure S3) adopts a favorable trans conformation, which is not the case in the Structure 2. For all these reasons, the Structure 1 was selected for further investigation on the on-state system. 


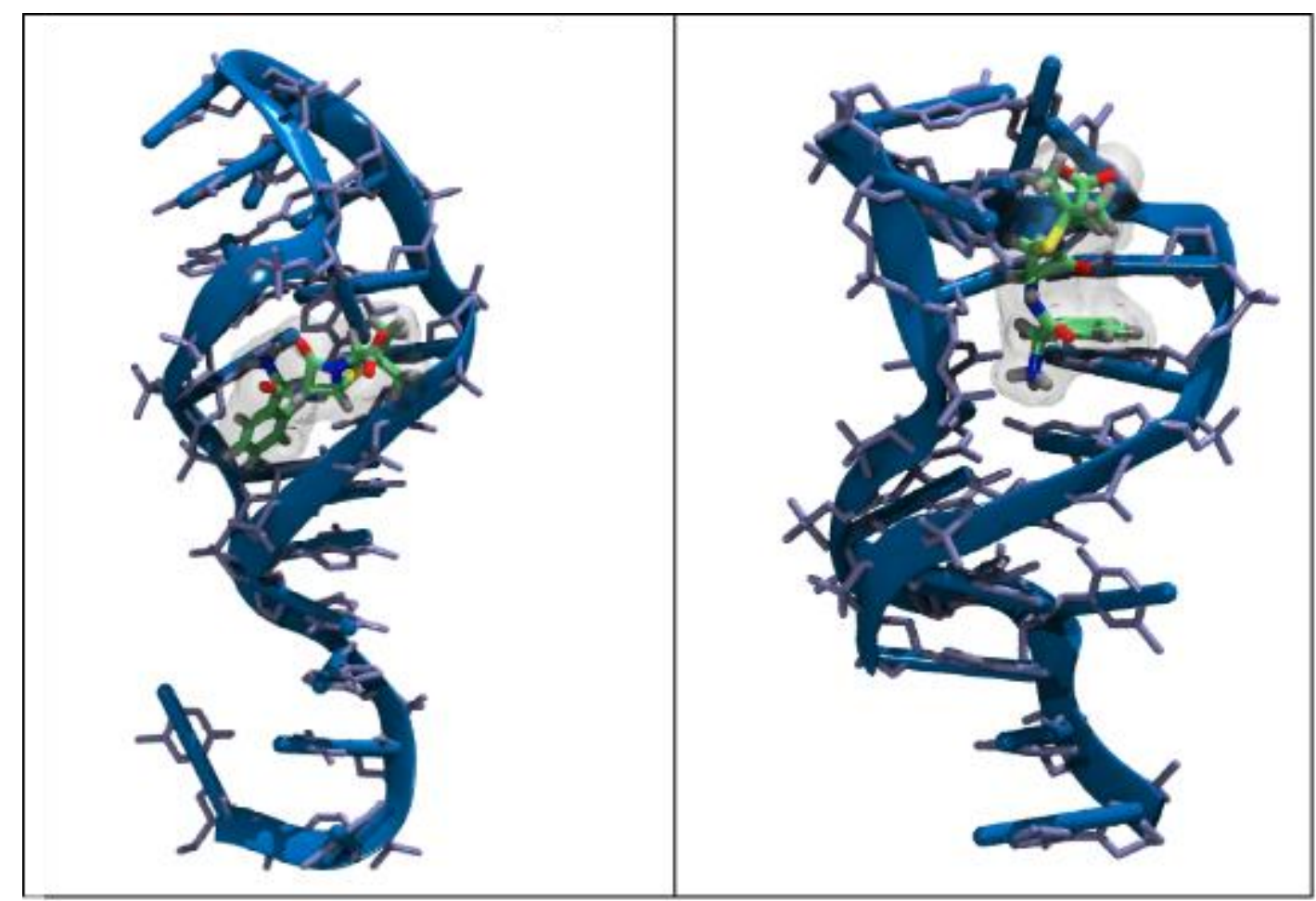

Figure 5. Aptamer-AMP structures. Left: Structure 1; Right: Structure 2. Nucleic acids are displayed in blue whereas the AMP ligand is colored by atom types (Carbon atoms in green, Nitrogen atoms in blue, Oxygen atoms in red, Sulphur atoms in yellow, Hydrogens in grey), its molecular surface is displayed in transparency.

\subsubsection{Addition of the AMP-aptamer complex to the on-state system}

The final AMP-aptamer complex was manually linked to DNA leg (see Scheme 1c). An MD simulation of $100 \mathrm{~ns}$ was run and the resulting structure was attached to the gold/MCH system by a phosphate linker. The other DNA leg which is dehybridized from the bridge was investigated by MD simulations in solution for $100 \mathrm{~ns}$. The resulting most representative structure was then attached to the $\mathrm{gold} / \mathrm{MCH}$ system by a phosphate linker. The surface with these two attachments constitutes the on-state system (Figure 3, right), which was finally investigated by MD simulations in duplicate.

\subsection{MD simulations of the full systems}

For both states (on- and off-), we monitored the kinetics, potential, and overall energies along the trajectory, as well as the densities, pressures or volumes, and temperatures. Root mean square deviations (RMSD) curves were produced for the nucleic acids, along the $100 \mathrm{~ns}$ trajectories, to observe global molecular movements. These curves, available on supplementary materials, reach a plateau indicating, this way, that the systems are 
structurally stable. In other words, no major distortion on the DNA structures grafted onto the surface was observed.

During MD trajectories visualization no collapse of the DNA structure onto the surface was observed. This indicates the reliability of the self-assembled monolayer which is usually used to prevent undesirable adsorption of biomolecules onto gold surfaces. In addition, we found that the DNA base pairs remain associated during the whole simulations emphasizing that the choose sequences are accurate. The third observation made focused on the ampicillin which remains bound in the on-state system demonstrating the high affinity of the aptameric sequence. Another important result is, for the on-state simulation, the lack of re-hybridization of the DNA strands, reforming the offstate. Taken together, these structural information evince the self-consistency of our approach.

The Root Mean Square Fluctuation (RMSF) values were computed for all MD trajectories in order to investigate the variation of flexibility between the systems in solution and grafted onto the $\mathrm{MCH}$ gold surface. For the on-state, the fluctuations are less informative to compare since, as expected, the two grafted DNA structures make no specific interaction together (data not shown). For the off-state, comparison of RMSF values in solution and onto the surface led to a decrease of these ones, as illustrated on Figure 6. This is notably the case for the extremities which are directly linked onto the surface. Interestingly, a sharp decrease of flexibility is revealed for the central dehybridized DNA section when the system is grafted to the surface. This demonstrates that the impact of the linkage is not limited to the first nucleic acids residues but extended to the whole sequence and stress to the importance of taking this parameter into consideration when selecting an optimal DNA sequence. 


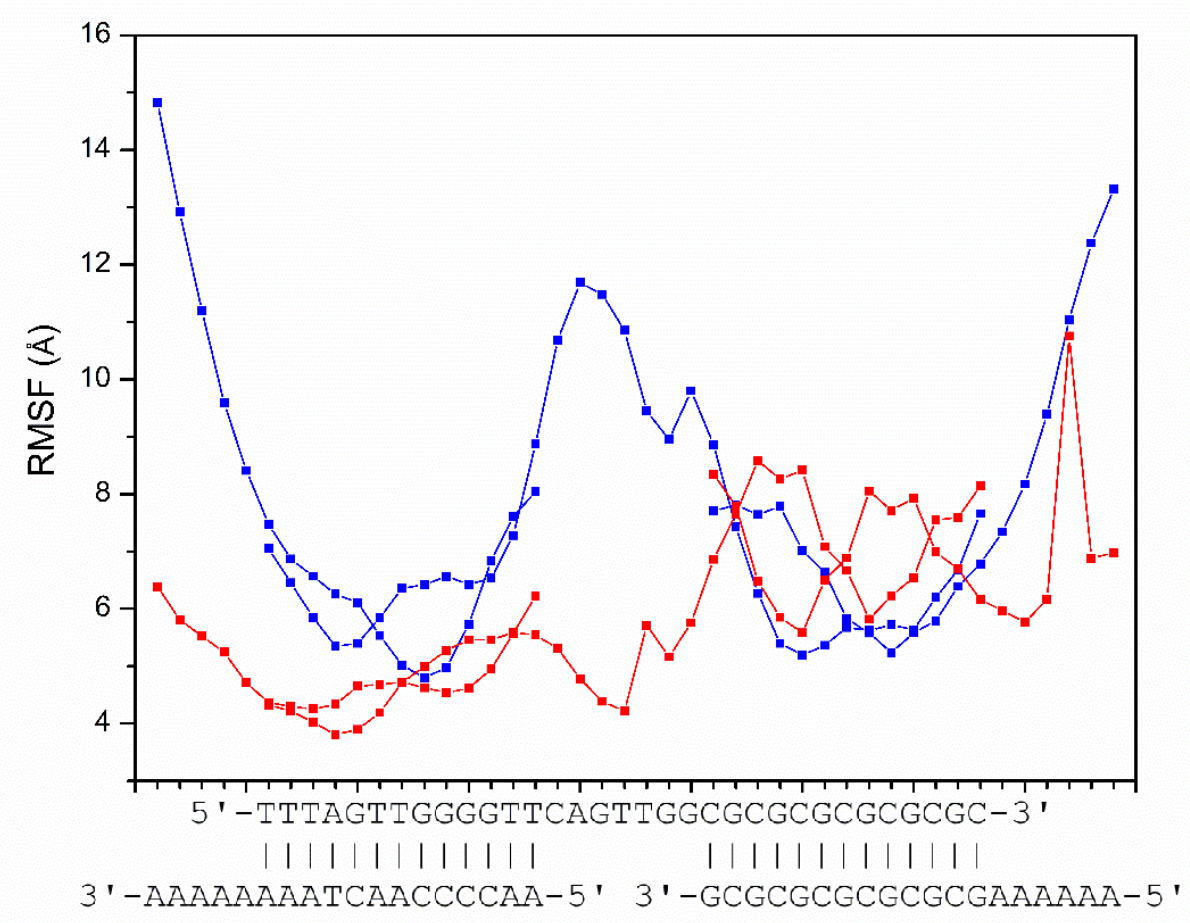

Figure 6. Graph of the RMSF values of the off-state (in $\AA$ ) obtained per base on the off-state system, in solution (blue) and grafted to the surface (red).

\subsection{Analysis of the changes in ion distribution}

To further investigate if the simulations are able to provide meaningful information on the detection ability of aptasensors based on dielectric properties change, we analyzed the ionic distribution over the last 100 ns of each replica by counting the ions over slabs of width $4 \AA$, parallel to the surface ${ }^{20}$. To obtain an average atom count, the total count was divided by the number of analyzed frames.

When a pure $\mathrm{MCH}$ monolayer is considered, $\mathrm{Na}^{+}$and $\mathrm{Cl}^{-}$concentration profiles have the same feature and are both homogeneous above a distance to the surface of approximately $2.5 \mathrm{~nm}$ (Figure S3 in Supporting Material), In the case of a mixed $\mathrm{MCH} / D N A$ monolayer. The $\mathrm{Cl}^{-}$concentration profile shows almost the same feature as for the pure $\mathrm{MCH}$ case whereas the $\mathrm{Na}^{+}$concentration strongly increases close to the surface for both the on- and off-state (see Fig. 7).

When comparing $\mathrm{Na}^{+}$concentration profiles between both states, a noticeable difference is observed in the ions concentration profile. The peaks on the concentration profile of 
$\mathrm{Na}$ + extend up to ca. $10 \mathrm{~nm}$ and $8 \mathrm{~nm}$ from the surface for the on- and the off-state respectively. There is a noticeable correlation between the localization profiles of $\mathrm{Na}^{+}$and phosphates in both states, indicating that the ionic distribution change is directly connected to the architecture conformation switch upon the AMP binding.
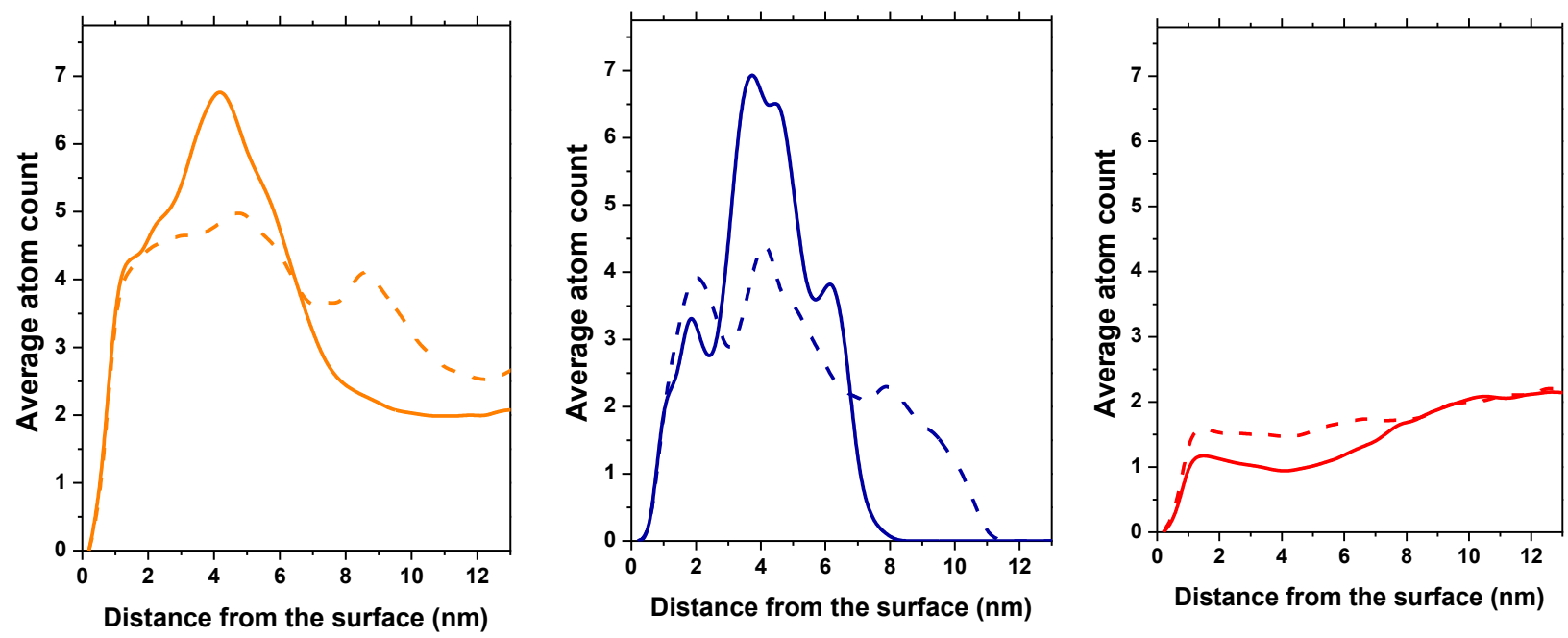

Figure 7. Graph of the average sodium ion count versus the distance to the surface in $\mathrm{nm}$. In orange, the $\mathrm{Na}^{+}$profile, in blue the phosphate (ROPO3R')- profile, and in red the $\mathrm{Cl}^{-}$profile. The continuous lines represent the off-state while the dotted lines represent the on-state.

\section{Conclusions}

We have proposed a novel DNA-based aptasensor structure for ampicillin (AMP) grafted on an $\mathrm{Au} / \mathrm{MCH}$ surface and we have studied a proposed structure by molecular modelling. We have performed a conformational study on the DNA system in solution in order to optimize its structure before grafting it to the surface to build our initial structure without AMP (off-state). We have elucidated the structure of an aptamer-ampicillin complex and used it to build our system while in presence of AMP (on-state). The expected conformational switch in the presence of AMP presents a change in ionic distribution at the vicinity of the surface (around $10 \mathrm{~nm}$ ) that might be detected by methods based on dielectric properties change such as electrochemical and field effect transistors (capacitance change) but also optical device (change in refracting index). This computational study opens the possibility to modulate, in-silico, the sequence of each partners in order to screen many different architectures and drive aptasensors design. 
As a prospect, and with the will to be as close as possible of real biosensors, especially transistors and electrochemical sensors, we plan to study the effects of electric charges present over the surface on the whole DNA structure and by consequence on the ionic distribution close to the surface.

\section{Acknowledgements}

Mario Araujo-Rocha gratefully acknowledges the financial support from the FET program within the Horizon 2020 Research and Innovation Program of the European Commission, under the HyPhOE FET-open project (grant agreement no.800926). ANR (Agence Nationale de la Recherche) and CGI (Commissariat à l'Investissement d'Avenir) are gratefully acknowledged for their financial support of this work through Labex SEAM (Science and Engineering for Advanced Materials and devices), ANR-10-LABX-096 and ANR-18-IDEX-0001. This work was granted access to the HPC resources of IDRIS under the allocation 2020-A0080711454 made by GENCI.

\section{References}

(1) The Aptamer Handbook: Functional Oligonucleotides and Their Applications; Klussmann, S., Ed.; Wiley-VCH: Weinheim, 2006.

(2) Song, S.; Wang, L.; Li, J.; Fan, C.; Zhao, J. Aptamer-Based Biosensors. TrAC Trends in Analytical Chemistry 2008, 27 (2), 108-117.

https://doi.org/10.1016/j.trac.2007.12.004.

(3) Jiang, D.; Yang, C.; Fan, Y.; Polly Leung, H.-M.; Inthavong, K.; Zhang, Y.; Li, Z.; Yang, M. Ultra-Sensitive Photoelectrochemical Aptamer Biosensor for Detecting E. Coli 0157:H7 Based on Nonmetallic Plasmonic Two-Dimensional Hydrated Defective Tungsten Oxide Nanosheets Coupling with Nitrogen-Doped Graphene Quantum Dots (DWO3•H2O@N-GQDs). Biosensors and Bioelectronics 2021, 183, 113214. https://doi.org/10.1016/j.bios.2021.113214.

(4) Zhang, K.; Li, H.; Wang, W.; Cao, J.; Gan, N.; Han, H. Application of Multiplexed Aptasensors in Food Contaminants Detection. ACS Sens. 2020, 5 (12), 37213738. https://doi.org/10.1021/acssensors.0c01740.

(5) Challier, L.; Miranda-Castro, R.; Barbe, B.; Fave, C.; Limoges, B.; Peyrin, E.; Ravelet, C.; Fiore, E.; Labbé, P.; Coche-Guérente, L.; Ennifar, E.; Bec, G.; Dumas, 
P.; Mavré, F.; Noël, V. Multianalytical Study of the Binding between a Small Chiral Molecule and a DNA Aptamer: Evidence for Asymmetric Steric Effect upon 3'versus 5'-End Sequence Modification. Anal. Chem. 2016, 88 (23), 11963-11971. https://doi.org/10.1021/acs.analchem.6b04046.

(6) Nangare, S. N.; Patil, P. O. Affinity-Based Nanoarchitectured Biotransducer for Sensitivity Enhancement of Surface Plasmon Resonance Sensors for In Vitro Diagnosis: A Review. ACS Biomater. Sci. Eng. 2021, 7 (1), 2-30. https://doi.org/10.1021/acsbiomaterials.0c01203.

(7) Yue, L.; Wang, S.; Zhou, Z.; Willner, I. Nucleic Acid Based Constitutional Dynamic Networks: From Basic Principles to Applications. J. Am. Chem. Soc. 2020, 142 (52), 21577-21594. https://doi.org/10.1021/jacs.0c09891.

(8) Morya, V.; Walia, S.; Mandal, B. B.; Ghoroi, C.; Bhatia, D. Functional DNA Based Hydrogels: Development, Properties and Biological Applications. ACS Biomater. Sci. Eng. 2020, 6 (11), 6021-6035. https://doi.org/10.1021/acsbiomaterials.0c01125.

(9) Liu, X.; Hu, M.; Wang, M.; Song, Y.; Zhou, N.; He, L.; Zhang, Z. Novel Nanoarchitecture of Co-MOF-on-TPN-COF Hybrid: Ultralowly Sensitive Bioplatform of Electrochemical Aptasensor toward Ampicillin. Biosensors and Bioelectronics 2019, 123, 59-68. https://doi.org/10.1016/j.bios.2018.09.089.

(10) Stojanovic, M. N.; de Prada, P.; Landry, D. W. Aptamer-Based Folding Fluorescent Sensor for Cocaine. J Am Chem Soc 2001, 123 (21), 4928-4931. https://doi.org/10.1021/ja0038171.

(11) Huizenga, D. E.; Szostak, J. W. A DNA Aptamer That Binds Adenosine and ATP. Biochemistry 1995, 34 (2), 656-665. https://doi.org/10.1021/bi00002a033.

(12) Da Costa, J. B.; Andreiev, A. I.; Dieckmann, T. Thermodynamics and Kinetics of Adaptive Binding in the Malachite Green RNA Aptamer. Biochemistry 2013, 52 (38), 6575-6583. https://doi.org/10.1021/bi400549s.

(13) Förster, U.; Weigand, J. E.; Trojanowski, P.; Suess, B.; Wachtveitl, J. Conformational Dynamics of the Tetracycline-Binding Aptamer. Nucleic Acids Res 2012, 40 (4), 1807-1817. https://doi.org/10.1093/nar/gkr835.

(14) Schwalbe, H.; Buck, J.; Fürtig, B.; Noeske, J.; Wöhnert, J. Structures of RNA Switches: Insight into Molecular Recognition and Tertiary Structure. Angew Chem Int Ed Engl 2007, 46 (8), 1212-1219. https://doi.org/10.1002/anie.200604163.

(15) Costa, D.; Garrain, P.-A.; Baaden, M. Understanding Small BiomoleculeBiomaterial Interactions: A Review of Fundamental Theoretical and Experimental 
Approaches for Biomolecule Interactions with Inorganic Surfaces. Journal of Biomedical Materials Research Part A 2013, 101A (4), 1210-1222. https://doi.org/10.1002/jbm.a.34416.

(16) Ozboyaci, M.; Kokh, D. B.; Corni, S.; Wade, R. C. Modeling and Simulation of Protein-Surface Interactions: Achievements and Challenges. Q Rev Biophys 2016, 49, e4. https://doi.org/10.1017/S0033583515000256.

(17) Thyparambil, A. A.; Bazin, I.; Guiseppi-Elie, A. Molecular Modeling and Simulation Tools in the Development of Peptide-Based Biosensors for Mycotoxin Detection: Example of Ochratoxin. Toxins (Basel) 2017, 9 (12). https://doi.org/10.3390/toxins9120395.

(18) Luna, R.-S.; James, G.-P.; Esteban, M.-M.; Francisco, I. In-Silico Design of Peptide Receptor for Carboxyhemoglobin Recognition. Informatics in Medicine Unlocked 2019, 14, 1-5. https://doi.org/10.1016/j.imu.2019.01.003.

(19) Rodriguez-Salazar, L.; Guevara-Pulido, J.; Cifuentes, A. In Silico Design of a Peptide Receptor for Dopamine Recognition. Molecules 2020, 25 (23), 5509. https://doi.org/10.3390/molecules25235509.

(20) Choodet, C.; Toomjeen, P.; Phanchai, W.; Matulakul, P.; Thanan, R.; Sakonsinsiri, C.; Puangmali, T. Combined in Silico and in Vitro Study of an Aptasensor Based on Citrate-Capped AuNPs for Naked-Eye Detection of a Critical Biomarker of Oxidative Stress. RSC Adv. 2019, 9 (31), 17592-17600. https://doi.org/10.1039/C9RA01497G.

(21) Ruan, M.; Seydou, M.; Noel, V.; Piro, B.; Maurel, F.; Barbault, F. Molecular Dynamics Simulation of a RNA Aptasensor. J. Phys. Chem. B 2017, 121 (16), 4071-4080. https://doi.org/10.1021/acs.jpcb.6b12544.

(22) Martin, J. A.; Chushak, Y.; Chávez, J. L.; Hagen, J. A.; Kelley-Loughnane, N. Microarrays as Model Biosensor Platforms to Investigate the Structure and Affinity of Aptamers. Journal of Nucleic Acids 2016, 2016, e9718612. https://doi.org/10.1155/2016/9718612.

(23) Sischo, W. M. Quality Milk and Tests for Antibiotic Residues. J Dairy Sci 1996, 79 (6), 1065-1073. https://doi.org/10.3168/jds.S0022-0302(96)76460-3.

(24) Centner, T. J. Recent Government Regulations in the United States Seek to Ensure the Effectiveness of Antibiotics by Limiting Their Agricultural Use. Environment International 2016, 94, 1-7. https://doi.org/10.1016/j.envint.2016.04.018. 
(25) Case, D. A.; Cheatham, T. E.; Darden, T.; Gohlke, H.; Luo, R.; Merz, K. M.; Onufriev, A.; Simmerling, C.; Wang, B.; Woods, R. J. The Amber Biomolecular Simulation Programs. J Comput Chem 2005, 26 (16), 1668-1688. https://doi.org/10.1002/jcc.20290.

(26) Zuker, M. Mfold Web Server for Nucleic Acid Folding and Hybridization Prediction. Nucleic Acids Res 2003, 31 (13), 3406-3415.

(27) Wang, J.; Wang, J.; Huang, Y.; Xiao, Y. 3dRNA v2.0: An Updated Web Server for RNA 3D Structure Prediction. Int J Mol Sci 2019, 20 (17). https://doi.org/10.3390/jms20174116.

(28) Ktari, N.; Fourati, N.; Zerrouki, C.; Ruan, M.; Seydou, M.; Barbaut, F.; Nal, F.; Yaakoubi, N.; Chehimi, M. M.; Kalfat, R. Design of a Polypyrrole MIP-SAW Sensor for Selective Detection of Flumequine in Aqueous Media. Correlation between Experimental Results and DFT Calculations. RSC Adv. 2015, 5 (108), 8866688674. https://doi.org/10.1039/C5RA16237H.

(29) Schrödinger Release 2021-1: Maestro, Schrödinger, LLC, New York, NY, 2021.

(30) Morris, G. M.; Huey, R.; Lindstrom, W.; Sanner, M. F.; Belew, R. K.; Goodsell, D. S.; Olson, A. J. AutoDock4 and AutoDockTools4: Automated Docking with Selective Receptor Flexibility. J Comput Chem 2009, 30 (16), 2785-2791. https://doi.org/10.1002/jcc.21256.

(31) Abramyan, T. M.; Snyder, J. A.; Yancey, J. A.; Thyparambil, A. A.; Wei, Y.; Stuart, S. J.; Latour, R. A. Parameterization of an Interfacial Force Field for Accurate Representation of Peptide Adsorption Free Energy on High-Density Polyethylene. Biointerphases 2015, 10 (2), 021002. https://doi.org/10.1116/1.4916361.

(32) Barbault, F.; Ren, B.; Rebehmed, J.; Teixeira, C.; Luo, Y.; Smila-Castro, O.; Maurel, F.; Fan, B.; Zhang, L.; Zhang, L. Flexible Computational Docking Studies of New Aminoglycosides Targeting RNA 16S Bacterial Ribosome Site. Eur J Med Chem 2008, 43 (8), 1648-1656. https://doi.org/10.1016/j.ejmech.2007.10.022.

(33) Gabr, M. T.; Barbault, F. First Dual Binder of MicroRNA-146a and Monomeric Tau: A Novel Approach for Multitargeted Therapeutics for Neurodegenerative Diseases. Chem. Commun. 2020, 56 (67), 9695-9698. https://doi.org/10.1039/D0CC04249H. (34) Morris, G. M.; Goodsell, D. S.; Halliday, R. S.; Huey, R.; Hart, W. E.; Belew, R. K.; Olson, A. J. Automated Docking Using a Lamarckian Genetic Algorithm and an Empirical Binding Free Energy Function. Journal of Computational Chemistry 1998, 19 (14), 1639-1662. https://doi.org/10.1002/(SICl)1096987X(19981115)19:14<1639::AID-JCC10>3.0.CO;2-B. 
(35) Teixeira, C.; Serradji, N.; Maurel, F.; Barbault, F. Docking and 3D-QSAR Studies of BMS-806 Analogs as HIV-1 Gp120 Entry Inhibitors. Eur J Med Chem 2009, 44 (9), 3524-3532. https://doi.org/10.1016/j.ejmech.2009.03.028.

(36) D. A. Case et al., Amber 20. University of California, San Francisco, 2020.

(37) Nam, K.; Gao, J.; York, D. M. An Efficient Linear-Scaling Ewald Method for LongRange Electrostatic Interactions in Combined QM/MM Calculations. J. Chem. Theory Comput. 2005, 1 (1), 2-13. https://doi.org/10.1021/ct049941i.

(38) Onufriev, A. V.; Izadi, S. Water Models for Biomolecular Simulations. WIREs Computational Molecular Science 2018, 8 (2), e1347. https://doi.org/10.1002/wcms.1347.

(39) Refinement of the Cornell et al. Nucleic Acids Force Field Based on Reference Quantum Chemical Calculations of Glycosidic Torsion Profiles | Journal of Chemical Theory and Computation https://pubs.acs.org/doi/10.1021/ct200162x (accessed 2021 -05 -06).

(40) Assessment of GAFF2 and OPLS-AA General Force Fields in Combination with the Water Models TIP3P, SPCE, and OPC3 for the Solvation Free Energy of Druglike Organic Molecules | Journal of Chemical Theory and Computation https://pubs.acs.org/doi/10.1021/acs.jctc.8b01039 (accessed 2021 -05-06).

(41) Jakalian, A.; Bush, B. L.; Jack, D. B.; Bayly, C. I. Fast, Efficient Generation of HighQuality Atomic Charges. AM1-BCC Model: I. Method. Journal of Computational Chemistry 2000, 21 (2), 132-146. https://doi.org/10.1002/(SICl)1096987X(20000130)21:2<132::AID-JCC5>3.0.CO;2-P.

(42) Jakalian, A.; Jack, D. B.; Bayly, C. I. Fast, Efficient Generation of High-Quality Atomic Charges. AM1-BCC Model: II. Parameterization and Validation. J Comput Chem 2002, 23 (16), 1623-1641. https://doi.org/10.1002/jcc.10128.

(43) Barbault, F.; Maurel, F. Is Inhibition Process Better Described with MD(QM/MM) Simulations? The Case of Urokinase Type Plasminogen Activator Inhibitors. Journal of Computational Chemistry 2012, 33 (6), 607-616. https://doi.org/10.1002/jcc.21983.

(44) Ryckaert, J.-P.; Ciccotti, G.; Berendsen, H. J. C. Numerical Integration of the Cartesian Equations of Motion of a System with Constraints: Molecular Dynamics of n-Alkanes. Journal of Computational Physics 1977, 23 (3), 327-341. https://doi.org/10.1016/0021-9991(77)90098-5. 
(45) Humphrey, W.; Dalke, A.; Schulten, K. VMD: Visual Molecular Dynamics. Journal of Molecular Graphics 1996, 14 (1), 33-38. https://doi.org/10.1016/02637855(96)00018-5.

(46) PTRAJ and CPPTRAJ: Software for Processing and Analysis of Molecular Dynamics Trajectory Data | Journal of Chemical Theory and Computation https://pubs.acs.org/doi/10.1021/ct400341p (accessed 2021 -05 -06).

(47) Kollman, P. A.; Massova, I.; Reyes, C.; Kuhn, B.; Huo, S.; Chong, L.; Lee, M.; Lee, T.; Duan, Y.; Wang, W.; Donini, O.; Cieplak, P.; Srinivasan, J.; Case, D. A.; Cheatham, T. E. Calculating Structures and Free Energies of Complex Molecules: Combining Molecular Mechanics and Continuum Models. Acc. Chem. Res. 2000, 33 (12), 889-897. https://doi.org/10.1021/ar000033j.

(48) Li, A.; Barbault, F.; Maurel, F.; Delamar, M.; Wang, B. Interaction Mode and Selectivity of the 2PU Inhibitor with the CDK4 and CDK2 Cyclin-Dependant Kinases: A Molecular Dynamics Study. Journal of Molecular Structure:

THEOCHEM 2008, 849 (1-3), 62-75. https://doi.org/10.1016/j.theochem.2007.10.015.

(49) Gourmala, C.; Luo, Y.; Barbault, F.; Zhang, Y.; Ghalem, S.; Maurel, F.; Fan, B. Elucidation of the LewisX-LewisX Carbohydrate Interaction with Molecular Dynamics Simulations: A Glycosynapse Model. Journal of Molecular Structure: THEOCHEM 2007, 821 (1-3), 22-29. https://doi.org/10.1016/j.theochem.2007.06.027.

(50) Aixiao, L.; François, M.; Florent, B.; Michel, D.; Baoshan, W.; Xiang, Z.; Ping, W. Molecular Modeling Study of Binding Site Selectivity of TQMP to G-Quadruplex DNA. European Journal of Medicinal Chemistry 2010, 45 (3), 983-991. https://doi.org/10.1016/j.ejmech.2009.11.040.

(51) Song, K.-M.; Jeong, E.; Jeon, W.; Cho, M.; Ban, C. Aptasensor for Ampicillin Using Gold Nanoparticle Based Dual Fluorescence-Colorimetric Methods. Anal Bioanal Chem 2012, 402 (6), 2153-2161. https://doi.org/10.1007/s00216-011-5662-3.

(52) Jeddi, I.; Saiz, L. Three-Dimensional Modeling of Single Stranded DNA Hairpins for Aptamer-Based Biosensors. Scientific Reports 2017, 7 (1), 1178. https://doi.org/10.1038/s41598-017-01348-5. 
Table S1: molecular docking results.

$\begin{array}{lcc}\text { Structure } & \text { DG in kcal } / \mathrm{mol} & \% \text { cluster } \\ \text { Aptamer 1 } & -7.3 & 22 \% \\ \text { Aptamer 2 } & -8.8 & 27 \% \\ \text { Aptamer 3 } & -7.6 & 28 \% \\ \text { Aptamer 4 } & -8.5 & 30 \% \\ \text { Aptamer 5 } & -7.8 & 55 \%\end{array}$

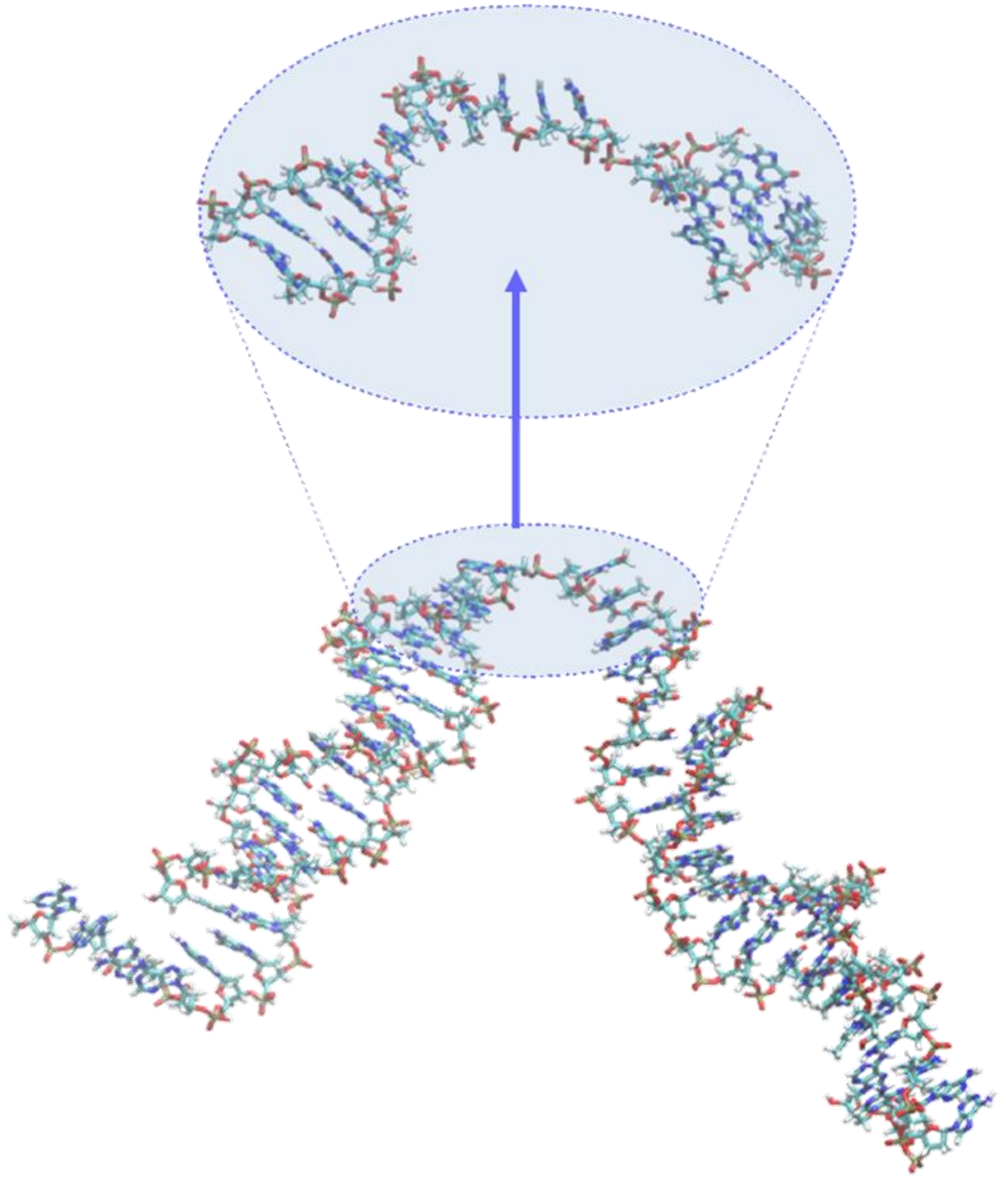


Figure S1: Schematic for the transferring of the parameters found for b) system to a) system described on Schematic 1 as restraints.

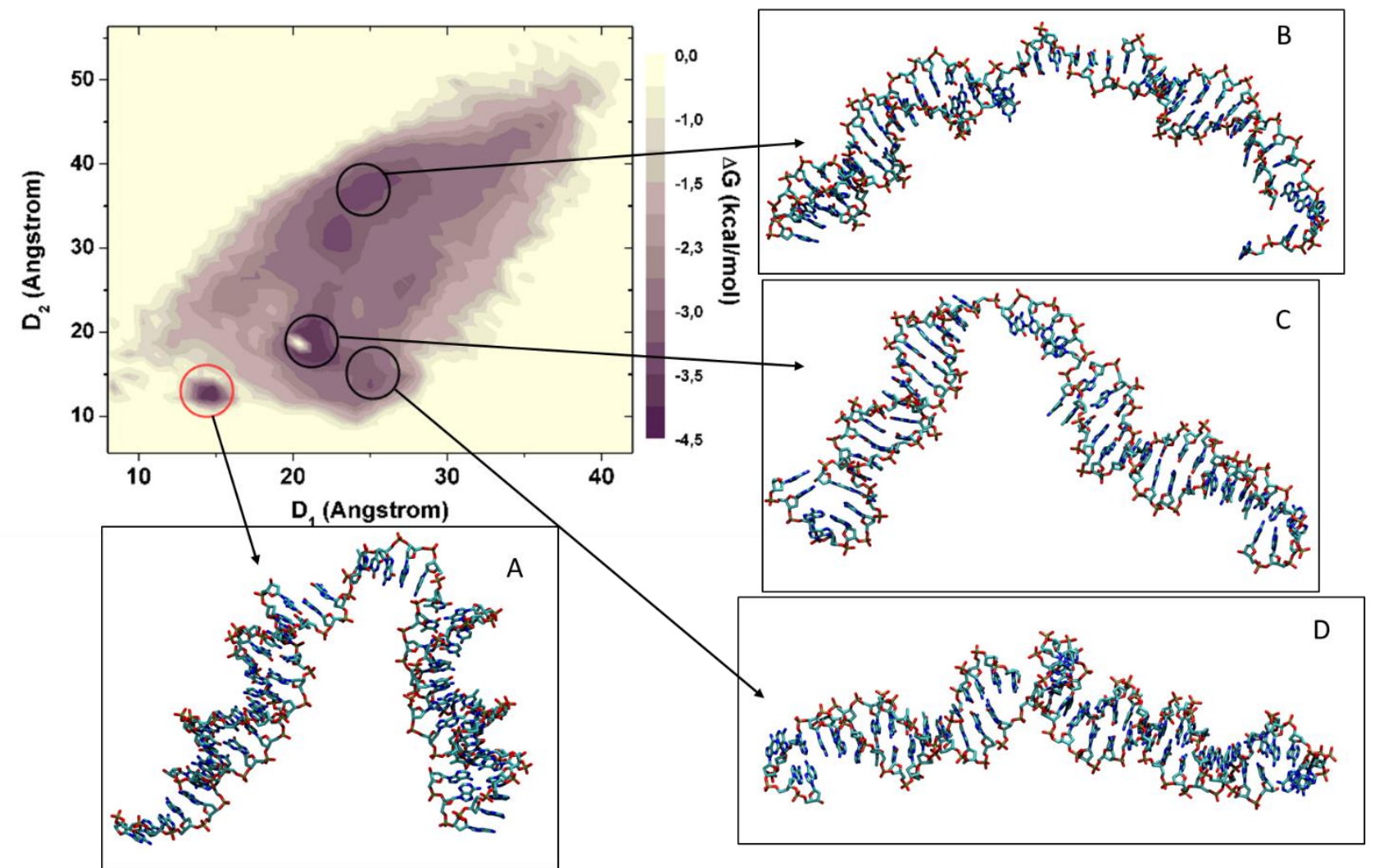

Figure S2: Final structures obtained for a) system after applying restraints indicated in Figure 2 and relaxation.

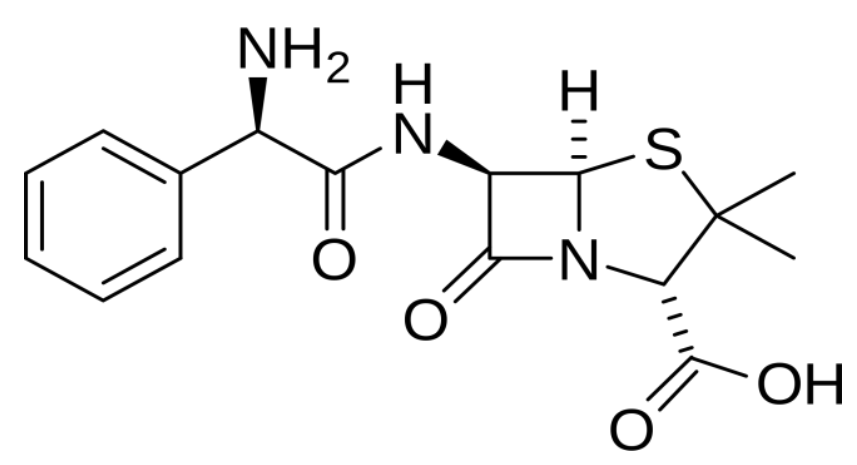

Figure S3: Molecular structure of AMP. 


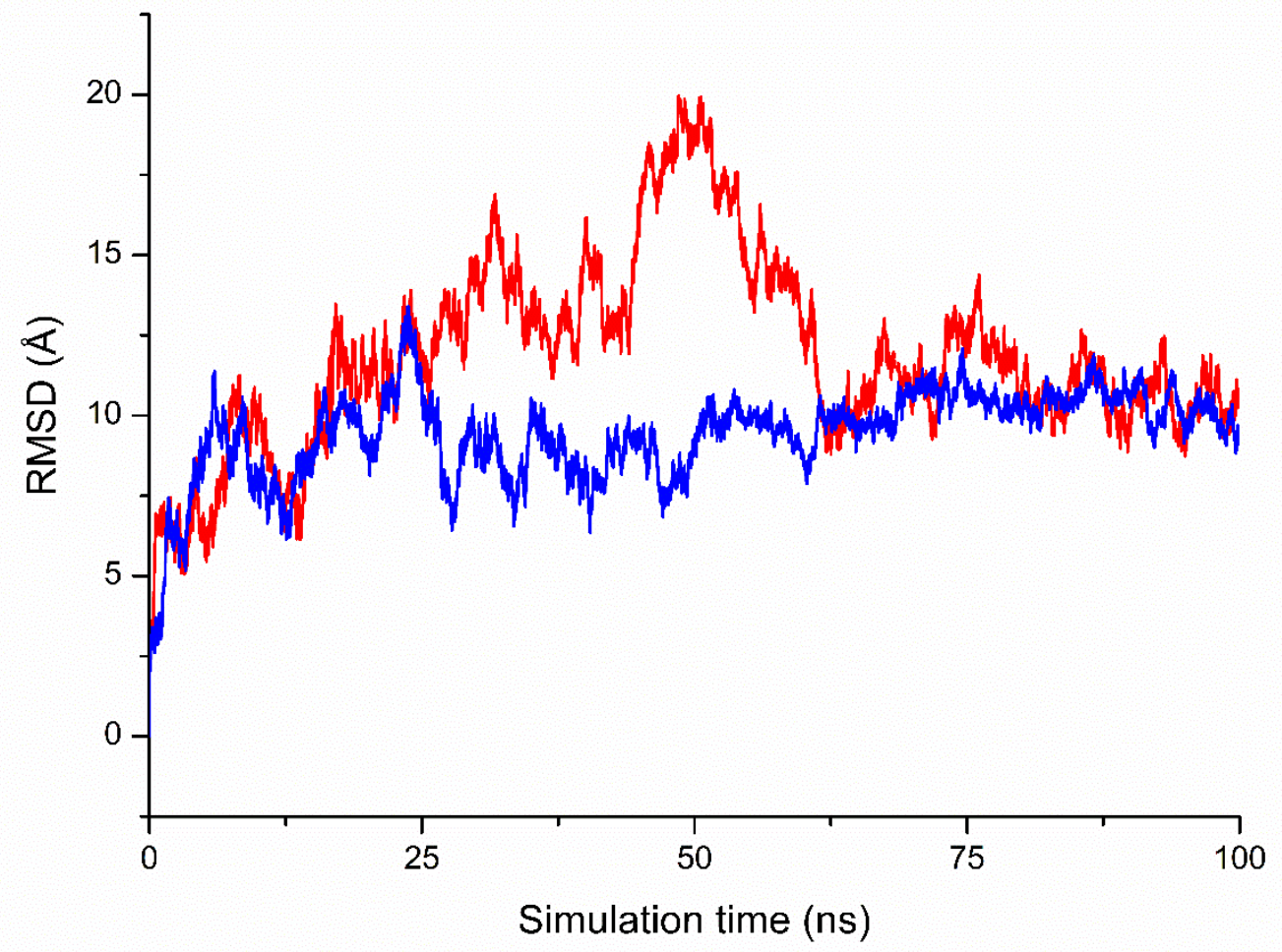

Figure S4: RMSD values (in Á) (all atoms) for the on-state (red) and the off-state (blue) DNA structures over $100 \mathrm{~ns}$ of simulation time with the first frame as reference.

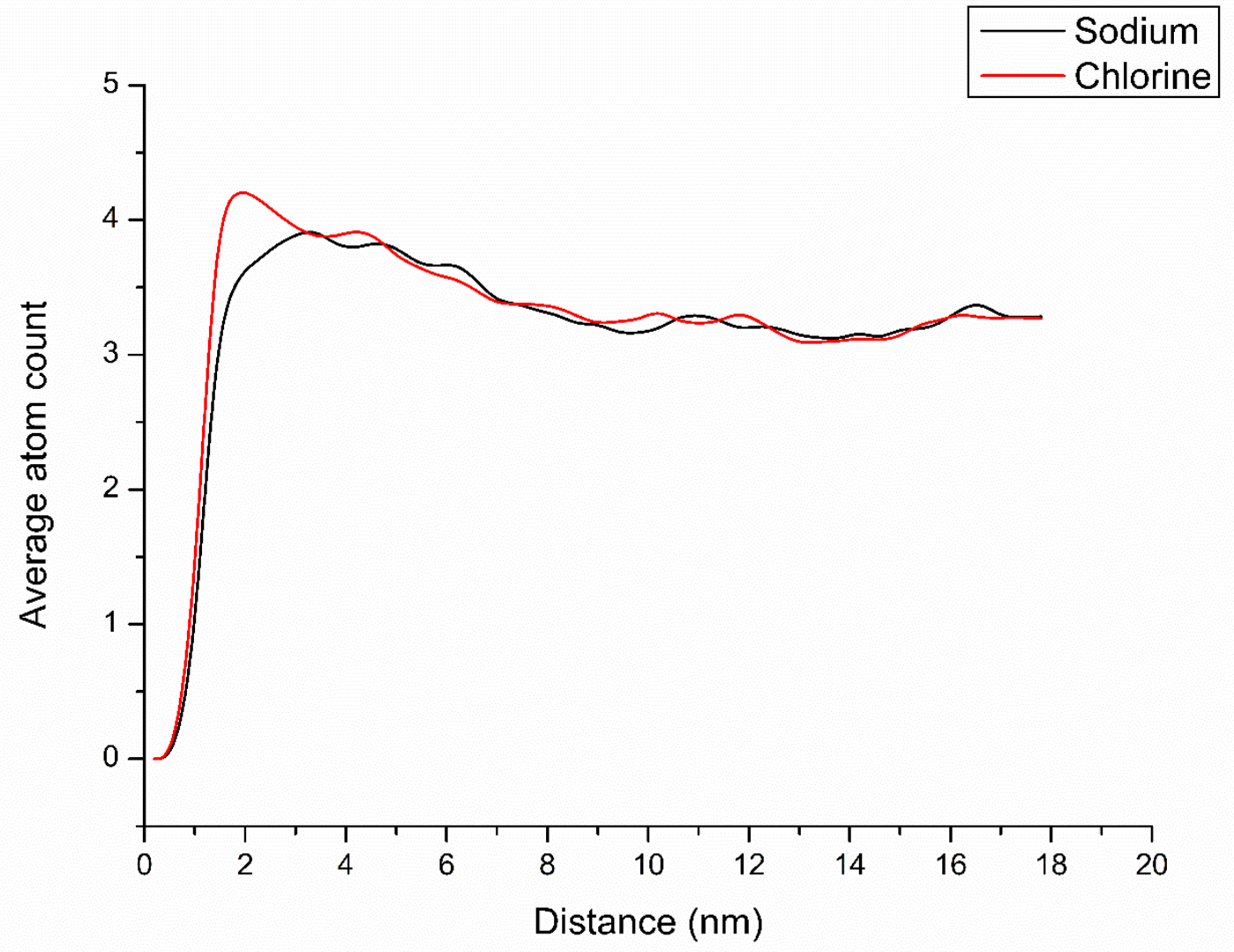

Figure S5: Ionic profile of the $\mathrm{NaCl} 160 \mathrm{mM}$ solution over an $\mathrm{Au} / \mathrm{MCH}$ surface without DNA. 